\title{
Obesity and the Endocannabinoid System: Circulating Endocannabinoids and Obesity
}

\author{
Isabelle Matias • Blandine Gatta-Cherifi • Daniela Cota
}

Published online: 17 August 2012

(C) Springer Science+Business Media, LLC 2012

\begin{abstract}
The endocannabinoid system is critically involved in the regulation of energy balance and represents a potential pharmacotherapeutic target for obesity and type 2 diabetes. New knowledge on the role of this system in humans has been obtained by measuring circulating endocannabinoids in plasma and serum, as well as in cerebrospinal fluid and saliva. Studies over the past few years have in particular provided information on the existing relationship between these biological measurements and the obese phenotype, feeding behavior or responses to body weight loss.
\end{abstract}

\section{Matias}

Group "Endocannabinoids and Neuroadaptation", INSERM,

Neurocentre Magendie, Physiophatologie de la Plasticité

Neuronale, U862,

3000 Bordeaux, France

e-mail: isabelle.matias@inserm.fr

B. Gatta-Cherifi • D. Cota

Group“Energy Balance and Obesity”, INSERM, Neurocentre

Magendie, Physiophatologie de la Plasticité Neuronale, U862,

33000 Bordeaux, France

B. Gatta-Cherifi

e-mail: blandine.gatta-cherifi@chu-bordeaux.fr

I. Matias · B. Gatta-Cherifi • D. Cota

Neurocentre Magendie, Physiopathologie de la Plasticité

Neuronale, U862, University of Bordeaux,

33000 Bordeaux, France

B. Gatta-Cherifi

Endocrinology Department, Haut-Lévêque Hospital,

33604 Pessac, France

D. Cota $(\bowtie)$

Group "Energy Balance and Obesity", INSERM U862,

Neurocentre Magendie,

146 Rue Léo Saignat,

33077 Bordeaux, France

e-mail: daniela.cota@inserm.fr
Although the functional significance of circulating endocannabinoids has yet to be proven, recent evidence suggests that they might be more than the simple by-product of tissular endocannabinoids spillover. This review outlines recent advances made in determining the potential relevance of circulating endocannabinoids as markers of obesity and metabolic disease.

Keywords Obesity · Circulating endocannabinoids · Quantification · Plasma · Saliva · Cerebrospinal fluid . Food intake - Weight loss

\section{Introduction}

Obesity and its associated metabolic disorders, such as hyperlipidemia and type 2 diabetes, have become a major public health threat worldwide [1-4].

In the hope of identifying appropriate molecular targets for efficient anti-obesity treatments, scientific research efforts have lately intensely focused on the unraveling of the biological mechanisms regulating food intake and body weight. In this context, the endocannabinoid system (ECS) has been recently recognized to have a major role in energy balance regulation, by affecting both central nervous system (CNS) and peripheral mechanisms involved in such physiological function, and its dysregulation is a landmark feature of obesity $[5,6]$.

The ECS comprises two distinct membrane receptors, the cannabinoid receptor type 1 (CB1) and type 2 (CB2), specific ligands named endocannabinoids and enzymes responsible of ligand biosynthesis and degradation [7, 8]. Extensive evidence in the literature suggests that endocannabinoid-dependent $\mathrm{CB} 1$ receptor activation is a powerful orexigenic signal and that, conversely, inhibition of $\mathrm{CB} 1$ receptor signaling is beneficial for the treatment of obesity and related metabolic 
diseases $[5,6]$. Mice lacking CB1 (CB1-KO) are lean and resistant to diet-induced obesity $[9,10]$. Accordingly, synthetic compounds like the $\mathrm{CB} 1$ inverse agonist rimonabant have shown potential as anti-obesity treatment in both animal models and obese patients [5, 6]. However, due to the important psychiatric side effects, the initial enthusiasm for the clinical use of rimonabant (commercial name Acomplia) faded away and the drug was subsequently withdrawn from the European market in January 2009 (http://www.ema.europa.eu/ema/). This clearly suggests that further knowledge about the broad mode of action of the ECS is required in order to better understand the functions of this system in human physiopathology and therefore allow targeting the system in a more selective and specific way.

In humans, particularly taking into account the difficulty to access biopsies and other tissue samples, the study of the ECS has recently focused on the assessment of circulating endocannabinoids and on their relationship with the obese phenotype.

Endocannabinoids are polyunsaturated fatty acids derivatives. So far, at least five endocannabinoids have been identified, of which the $N$-ethanolamide of arachidonic acid, also known as anandamide (AEA), and the glyceryl ester of arachidonic acid or 2-arachidonoylglycerol (2-AG) are the best characterized [11, 12].

In the CNS, endocannabinoids act as retrograde signals at the $\mathrm{CB} 1$ receptor to decrease neurotransmitter release at nerve terminals [13]. However, while their function as chemical neuromediators is very well defined, less is known about the biological significance, if any, of circulating endocannabinoids. Elevated circulating (in plasma or serum) AEA and 2AG levels have been described in obese humans and, depending on the study, positively correlated with different measures of adiposity, including waist circumference, body fat percentage and body mass index (BMI) (Table 1). However, whether such measurements simply represent a passive spillover from tissues in which the ECS is overactive [14] or bring also functional information is currently a matter of debate. Here, we provide a critical overview on the recent advancements made in understanding the function of circulating endocannabinoids in human obesity.

\section{Endocannabinoids Quantification}

One first aspect that needs to be considered when assessing endocannabinoids levels is that the quantification of these derivatives of arachidonic acid is not trivial. In addition, given the low concentration of endocannabinoids, particularly in the blood, growing efforts have been made in order to establish accurate sample preparation and quantification $[15,16 \bullet]$. Nevertheless, endocannabinoids quantification still remains an analytical challenge, and differences in their levels among different studies often mirror differences in the sampling, extraction and quantification method used. Even though the type of chromatography coupled to mass spectrometry seems to not significantly affect the measurements, since it has been shown that plasma AEA levels measured by liquid chromatography coupled to tandem mass spectrometry (LC-MS/MS) correlated closely with those measured by gas chromatography coupled to tandem mass spectrometry (GC-MS/MS) $[15,16 \bullet]$. However, sampling and sample treatment are crucial steps for correct endocannabinoids analysis, in particular considering that the half-life of endocannabinoids in vivo is of the order of few minutes $[15,16 \bullet]$. Consequently, biological samples, such as blood, should be immediately put on ice at time of collection, rapidly centrifuged and plasma (or serum) quickly frozen and stored at $-80^{\circ} \mathrm{C}$ until analysis $[16 \bullet, 17 \bullet]$. During the sample extraction, it is also crucial to pay attention to endocannabinoid absorption on the plastic ware, to the extraction solvent used and to the evaporation step under nitrogen. Even if similar extraction yield is obtained using the classical chloroform:methanol technique and toluene, the toluene solvent reduces the 2-AG isomerization and consequently may affect $1-\mathrm{AG}$ levels, which likely derive from 2-AG isomerization [18]. Finally, using the same technique and methodological steps also endocannabinoidrelated $N$-acylethanolamines such as oleoylethanolamide (OEA) and palmitoylethanolamide (PEA) can be quantified.

\section{Effect of Gender and Age on Endocannabinoid Levels}

Apart from the method followed for the quantification of endocannabinoids, other variables that can affect endocannabinoids measurement and that have only recently started to be taken into account include factors that are known to critically influence biological systems, such as the age of the subjects and their gender.

A first study by Bluher and colleagues in 2006 reported that plasma AEA levels were higher in women as compared to men, but not different between lean and obese cohorts [19]. Instead, no sex differences were found for plasma 2AG concentrations, although 2-AG correlated with abdominal visceral fat area, body mass index (BMI) and fasting insulin [19]. Interestingly, in this study a correlation between plasma 2-AG concentrations and age was observed only in women [19].

Differently, Fanelli and collaborators were unable to observe gender differences in plasma AEA levels in a large cohort of healthy, normal weight subjects [16•]. Instead, they described significantly higher plasma $2-\mathrm{AG}$ levels in men rather than women and consequently estimated genderspecific reference intervals for endocannabinoids and their associated $N$-acylethanolamines OEA and PEA [16•]. AEA and OEA showed a tendency to increase with age in women, but only PEA reached statistical significance; whereas 2-AG 
Table 1 Circulating endocannabinoids and related $N$-acylethanolamines in humans and their association with anthropometric and metabolic parameters

\begin{tabular}{llll}
\hline Subjects & Results & Correlations
\end{tabular}

20 obese women (W) and 20 lean W

$15 \mathrm{~W}$ healthy control

$11 \mathrm{~W}$ with anorexia nervosa

$8 \mathrm{~W}$ with bulimia nervosa

$11 \mathrm{~W}$ with BED

5 men $(\mathrm{M})$ and $3 \mathrm{~W}$ normal weight / $4 \mathrm{M}$ and $6 \mathrm{~W}$ obese type 2 diabetic

$10 \mathrm{M}$ and $10 \mathrm{~W}$ lean

$10 \mathrm{M}$ and $10 \mathrm{~W}$, subcutaneous obesity

$10 \mathrm{M}$ and $10 \mathrm{~W}$, visceral obesity

$62 \mathrm{M}$ $\checkmark$ plasma AEA \& 2-AG obese vs. lean

$\nearrow$ plasma AEA anorexic W vs healthy

$\nearrow$ plasma AEA BED W vs healthy
Positive plasma AEA with BMI

Positive plasma 2-AG with waist circumference

Negative plasma AEA with plasma leptin in healthy $\mathrm{W}$ and anorexic $\mathrm{W}$

7 plasma AEA \& 2-AG type 2 di- Not done abetic obese vs. normal weight

$\nearrow$ plasma AEA obese vs. lean

$\checkmark$ plasma $2-A G$ with visceral obesity vs.sub cutaneous obesity

No comparison between groups

Positive plasma 2-AG / BMI, \%fat mass \& visceral adipose tissue

Negative plasma AEA with intra abdominal
$6 \mathrm{~W}, 4 \mathrm{M}$ lean insulin sensitive

$6 \mathrm{~W}, 4 \mathrm{M}$ insulin resistant obese

$7 \mathrm{M}$ non diabetic and $12 \mathrm{M}$ diabetic

$49 \mathrm{M}$ before and after 1 year of lifestyle intervention

$17 \mathrm{M}, 31 \mathrm{~W}$ normal weight and $43 \mathrm{M}, 53 \mathrm{~W}$ obese

20 obese patients $(18 \mathrm{M}, 2 \mathrm{~F})$ with sleep apnea and 57 overweight healthy controls (28 M, 29 F)

21 controls, 26 overweight and 30 obese subjects

$\nearrow$ plasma AEA obese vs controls

27 M (12 Caucasians, 11 American Indians, 4 African Americans)

$76 \mathrm{~W}$ and $45 \mathrm{M}$ normal weight subjects

$2 \mathrm{M}$ and $10 \mathrm{~W}$ normal weight and $4 \mathrm{M}, 8 \mathrm{~W}$ insulin resistant obese

$2 \mathrm{M}$ and $10 \mathrm{~W}$ normal weight and $4 \mathrm{M}, 8 \mathrm{~W}$ insulin resistant obese $\nearrow$ plasma AEA insulin resistant vs. insulin sensitive

Plasma 2-AG not different

No differences between non diabetic and diabetic patients

$\checkmark$ plasma AEA after intervention

$\checkmark$ plasma 2-AG after intervention

$\nearrow$ (trend) plasma AEA Obese vs. Normal weight

plasma OEA in sleep apnea group plasma OEA in sleep apnea groun
No differences according races in plasma levels

$\nearrow \mathrm{CSF} 2-\mathrm{AG}$ in American Indians
7 plasma 2-AG in males compare to females

$\checkmark$ plasma PEA with age in females

plasma AEA and 2-AG in obese vs normal weight adipose tissue

Positive plasma 2-AG with BMI, waist circumference, triglycerides, fasting insulin

Negative plasma 2-AG withHDL Cholesterol and Adiponectin

If insulin sensitive subjects and insulin resistant subjects considered together:

Positive plasma AEA with BMI, waist circumference, triglycerides

Positive plasma 2-AG withtriglycerides

Positive plasma 2-AG with triglycerides

Positive \} \backslash \text { plasma } 2 \text { -AG with } \searrow \text { visceral adi- } pose tissue

Positive plasma AEA with FAAH 385A mutation

In the sleep apnea group:

Positive plasma OEA with respiratory distress index

Positive plasma AEA with BMI and HOMA

Positive plasma AEA with BMI, waist circumference, fasting insulin

Negative CSF AEA with BMI, waist circumference, fasting insulin

Positive CSF OEA with $24 \mathrm{~h}$ and sleep energy expenditure

Positive plasma AEA, PEA and OEA with each other

Positive plasma AEA with BMI, waist circumference and fasting insulin in females

Positive plasma 2-AG with age in females

Positive plasma AEA /BMI, waist circumference, fasting insulin

Positive plasma 2-AG /BMI, waist circumference

Positive saliva AEA and OEA with BMI, waist circumference, fasting insulin

Positive saliva 2-AG with waist circumference, fasting insulin

$B M I$ Body mass index, CSF Cerebrospinal fluid, FAAH Fatty acid amide hydrolase, HOMA Homeostatic model assessment 
displayed a positive correlation with age in women [16•]. No correlation was observed between 2-AG, OEA, PEA or OEA and age in men [16•]. Finally, AEA correlated with BMI, waist circumference, and fasting insulin in healthy women only, while 2-AG correlated only with triglycerides, independently of gender [16•].

The differences between the two abovementioned studies might be due to the fact that while Bluher and colleagues investigated small cohorts of obese patients, Fanelli and collaborators studied a large group of healthy, normal weight subjects. Although these findings are not conclusive, they lead to suggest that gender and age might indeed affect circulating endocannabinoids levels, as it would be expected taking also into account recent studies investigating the relationship of the ECS with gonadal hormones [20] and the role of this system in ageing [21].

\section{Endocannabinoids and Obesity}

As briefly mentioned before, several studies over the past few years have demonstrated that circulating endocannabinoids are elevated in obesity, as compared to normal weight, and positively correlated with different measures of adiposity, including waist circumference, body fat percentage and BMI (Table 1).

A first study published in 2005 showed that overweight/ obese women with binge eating disorder (BED) had increased plasma concentrations of AEA and normal plasma levels of 2-AG as compared to lean women [22]. Plasma AEA levels were significantly and positively correlated to body fat mass, but not to body weight, BMI or body lean mass [22]. In another study published that same year, it was reported that obese post-menopausal women had an increase of 35 and $52 \%$, respectively, in plasma levels of AEA and 2-AG as compared to normal weight women [23]. Increased plasma endocannabinoids and $N$-acylethanolamines levels were also described in overweight, type 2 diabetic men and women $[24,25]$. In addition, Bluher et al. and Coté et al. have shown that 2-AG plasma levels were positively correlated with abdominal obesity, while obese subjects characterized by subcutaneous adipose tissue accumulation had circulating endocannabinoids levels comparable to those of normal weight controls $[19,26]$. Plasma $2-A G$ was found to directly correlate with triglycerides and fasting insulin, while it inversely correlated with HDL cholesterol and adiponectin [26]. Besides, plasma 2-AG levels were associated with decreased insulin sensitivity and alterations in glucose metabolism, as suggested by their negative correlation with glucose infusion rate during clamp [19] and positive association with a significantly greater glycemic response during an oral glucose load [26]. More recent studies have reported that also plasma AEA levels correlate with metabolic parameters $[17 \bullet, 27 \bullet]$. In particular, we recently demonstrated that obese, insulin-resistant subjects had elevated plasma AEA and 2-AG levels, and that AEA levels were directly correlated with fasting insulin [17•]. Interestingly, insulin resistance has been proposed as one of the main events associated with the increased endocannabinoid tone observed in obesity [27•]. In fact, insulin infusion decreases AEA levels in humans, but it is unable to do so once insulin resistance has developed [27•].

Apart from insulin resistance, other conditions are often associated to obesity, including hypertension, cardiovascular disease and sleep apnea [28]. Recently, Quercioli and colleagues have described that increased plasma AEA and 2-AG levels are associated with coronary circulatory dysfunction in obese individuals, thus implying that endocannabinoid plasma levels might be a novel endogenous cardiovascular risk factor in obesity [29]. While, in a different study it was shown that subjects characterized by a greater BMI and sleep apnea also had increased serum OEA levels [30].

In addition to blood, endocannabinoids and related $\mathrm{N}$ acylethanolamines are quantifiable in the cerebrospinal fluid (CSF) and in saliva. In a recent study, Jumpertz and colleagues analyzed paired CSF and plasma samples in a group of obese subjects with diverse racial background, showing that plasma AEA concentrations were strongly and positively associated with all available measures of adiposity [31 $]$. In contrast, CSF AEA and PEA were negatively associated with measures of adiposity, while CSF OEA levels positively correlated with different measures of energy expenditure [31•]. Finally, AEA, OEA and PEA were significantly correlated with each other in CSF and plasma, respectively; however, there were no associations with 2-AG in either of the two compartments [31•]. These findings therefore imply that the peripheral (circulating in blood) and central (circulating in CSF) role of endocannabinoids and cognate $\mathrm{N}$ acylethanolamines may be very different, since peripheral, but not central, AEA levels were found to be most strongly associated with obesity [31•].

Finally, and in agreement with the increases described in plasma, we have recently demonstrated that salivary endocannabinoids and related $N$-acylethanolamines are increased in obese subjects as compared to normal weight controls and that their levels directly correlate with markers of adiposity and metabolic alteration [32•].

Thus, while it is evident that overweight and obesity, particularly the one associated to metabolic alterations such as insulin resistance and type 2 diabetes, are generally characterized by increased levels of endocannabinoids, more difficult is to understand whether such alteration and the underlying dysregulated activity of the ECS are the cause or the consequence of the body weight gain.

In this context, it is important to highlight that genetic studies have investigated the polymorphisms of CNR1 (coding for $\mathrm{CB} 1$ receptor) and FAAH (fatty acid amide hydrolase, 
one of the principal enzymes degrading endocannabinoids) in obese populations. In general, associations between polymorphisms in these genes and abdominal obesity [33], metabolic syndrome [34] or dyslipidemia [35] have been found in spite of some discrepancies. However, it must be kept in mind that such associations suggest only a partial and rather weak contribution to the obese phenotype and that they may influence obesity, and eventually response to treatment, only under specific diet conditions [36]. Interestingly, in 2010, Sipe and co-workers demonstrated that the FAAH $385 \mathrm{~A}$ polymorphism (a mutation leading to a decreased activity of the FAAH enzyme) was directly associated with elevated plasma levels of AEA and related $N$-acylethanolamines OEA and PEA in obese subjects. This evidence therefore supports the activation of the ECS due to the reported FAAH 385A polymorphism. Elevated levels of AEA and related $N$-acylethanolamines in carriers of the FAAH 385A polymorphism might therefore identify a phenotype at higher risk for the development of obesity [37•].

\section{Circulating Endocannabinoids and Weight Loss}

Only three studies have so far investigated the effect of weight loss on plasma endocannabinoid levels [23, 38, 39]. The first study was carried out on obese women in whom a $5 \%$ weight loss was obtained after 13-15 weeks of dietary weight reduction protocol and whose weight was then maintained stable for a subsequent period of 3 months [23]. Under these conditions, plasma endocannabinoids, which were measured during the body weight loss maintenance period, were unchanged [23]. Similarly, acute (5 days) or chronic ( 3 months) treatment of obese patients with sibutramine (serotonin-norepinephrine reuptake inhibitor) did not alter plasma endocannabinoid levels [38]. Conversely, a 1 year lifestyle intervention program including healthy eating and physical activity and leading to $7 \%$ weight loss and abdominal fat loss $(-8.0 \mathrm{~cm}$ of waist circumference) in obese men was associated to a significant decrease of both plasma 2-AG (-62.3\%) and AEA (-7.1\%) levels [39]. The decrease in plasma 2-AG levels in particular correlated with decreases in visceral adipose tissue and triacylglycerol levels, and with the increase in HDLcholesterol levels [39]. In addition, multivariate analyses demonstrated that decreases in 2-AG and visceral adipose tissue were independently associated with decreases in triacylglycerol levels [39].

Likely, the inconsistency across the different studies published so far on the relationship between body weight loss and plasma endocannabinoids depends on several factors, including the rate of body weight loss, the point in time when the endocannabinoids measurement was carried out with respect to when the body weight loss goal was reached and possibly the type of diet followed by the patients to induce and maintain body weight loss, since the composition of the diet can affect the endocannabinoids precursors' pool [40].

Finally, it should be mentioned that body weight loss might not only affect blood, but also salivary endocannabinoid levels. Indeed, a $5 \%$ body weight loss induced by a lifestyle intervention program led to a specific decrease of salivary AEA, with no changes in $2-\mathrm{AG}$ and other $N$-acylethanolamines [32•]. Thus, the assessment of salivary endocannabinoids and related compounds might be useful for phenotypic and eventually therapeutic purposes, in particular considering that salivary samples require a non-invasive procedure and are easy to collect.

\section{Endocannabinoids and Food Intake}

In animals, variations of endocannabinoids levels in tissues are in agreement with the general orexigenic role of the ECS. In particular, endocannabinoid levels increase in the hypothalamus, limbic system and small intestine of rodents with fasting, while decrease during refeeding [41, 42].

In humans, it has been shown that both in fasting and non-fasting states circulating AEA and other $N$-acylethanolamines levels were correlated with free fatty acid levels, suggesting that similar physiological stimuli might cause the release of both plasma $N$-acylethanolamines and free fatty acids in blood [43]. Matias and collaborators were the first to actually show that plasma levels of AEA and related $N$-acylethanolamines were decreased in healthy volunteers one hour after the consumption of a high-fat meal $[24,25]$, implying that circulating levels of these compounds might change in relation to food intake. A more recent study carried out by our group has actually shown that both normal-weight and obese subjects have a significant preprandial AEA peak, entailing that AEA might be a physiological meal initiator [17•]. We also reported that $1 \mathrm{~h}$ after the consumption of a calorically-balanced meal, AEA levels significantly decreased in normal-weight, whereas no significant changes were observed in obese subjects [17•]. Similarly, levels of the satiety hormone peptide YY (PYY) significantly increased in normal-weight subjects only. Postprandial AEA and PYY changes inversely correlated with waist circumference, and independently explained 20.7 and $21.3 \%$ of waist variance in the recruited subjects [17•]. Surprisingly, no meal-related changes were found for 2$\mathrm{AG}$, suggesting that AEA and 2-AG might have distinct roles in response to food intake.

Interestingly, Monteleone and colleagues recently showed that higher levels of plasma $2-A G$ and ghrelin, an orexigenic gastric hormone, are observed before the ad libitum consumption of highly palatable food, but not at time of consumption of an isocaloric amount of nonpalatable food, thus leading to conclude that when 
motivation to eat is generated by the availability of highly palatable food rather than hunger, a peripheral activation of these two signals occurs [44•].

The question however remains of where the observed plasma changes in AEA and 2-AG originate from. A first candidate would be the gastrointestinal tract, since not only this organ is directly exposed to the nutrients introduced through the diet, but it is also characterized by tissular endocannabinoid levels that are respectively increased and decreased by fasting and refeeding [41]. Differently from what is described for plasma endocannabinoids, salivary endocannabinoids did not change in relation to the consumption of a meal, implying that plasma and saliva endocannabinoids originate from separate compartments, as also suggested by the fact that the ECS, with its ligands, receptors and enzymes, localizes within the human salivary glands [32•]. A possibility is that salivary endocannabinoids might specifically affect food taste rather than respond to food intake, since the administration of AEA or 2-AG in mice increases gustatory nerve responses to sweet taste and CB1 receptors have been localized on taste bud cells expressing sweet taste receptors [45].

\section{Conclusions}

Although the origin of the endocannabinoids and related $\mathrm{N}$ acylethanolamines circulating in the blood is still debated, a series of studies over the past 7 years has highlighted the strong positive association between their levels and obesity. Recent investigations have also shown that endocannabinoids can be quantified in other biological compartments and that their measurement in the CSF and saliva might represent a useful tool for the study of the physiopathological role of the ECS in obesity. In addition, novel findings link food intake and food palatability to changes in plasma AEA and 2-AG levels, suggesting that these measurements might mirror rapid changes in ECS activity. Although exact location of these changes is currently unknown, one of the main targeted organs might be the gastrointestinal tract for the reasons illustrated above.

Overall, the evidence reviewed here although relevant, should be interpreted with caution since often the aforementioned studies were carried out in small cohorts, were lacking age- and gender-matched groups and were characterized by different extraction and quantification methods, leading to great variability in the levels of measured endocannabinoids and associated $N$-acylethanolamines in the different studies. We agree with Quarta and colleagues [6] about the need of reference intervals and of the standardization of the quantification procedures for the further use of endocannabinoid measurements for phenotypic and therapeutic purposes.
Acknowledgments D. Cota has received the following in grant support: NSERM; INSERM/contract interface; AQUITAINE Region; SFD (French Society diabetology); SFE (French society endocrinology; FP7EU-FP7 HEALTH-F2-2008-223713; CHU Bordeaux. B. Gatta-Cherifi has received SFD and SFE. Work by I. Matias was supported by FP7-EUFP7 HEALTH-F2-2008-223713.

Disclosure Conflicts of interest: I. Matias: none; B. Gatta-Cherifi: none; D. Cota: has been a consultant for Sanofi-Aventis US, 2006-2008.

\section{References}

Papers of particular interest, published recently, have been highlighted as:

- Of importance

1. Smyth S, Heron A. Diabetes and obesity: the twin epidemics. Nat Med. 2006;12:75-80.

2. Cooke D, Bloom S. The obesity pipeline: current strategies in the development of anti-obesity drugs. Nat Rev Drug Discov. 2006;5:919-31.

3. Sandoval DA, Obici S, Seeley RJ. Targeting the CNS to treat type 2 diabetes. Nat Rev Drug Discov. 2009;8(5):386-98.

4. Tahrani AA, Bailey CJ, Del Prato S, Barnett AH. Management of type 2 diabetes: new and future developments in treatment. Lancet. 2011;378:182-97.

5. Bermudez-Silva FJ, Cardinal P, Cota D. The role of the endocannabinoid system in the neuroendocrine regulation of energy balance. J Psychopharmacol. 2012;26:114-24.

6. Quarta C, Mazza R, Obici S, et al. Energy balance regulation by endocannabinoids at central and peripheral levels. Trends Mol Med. 2011;17:518-26.

7. Di Marzo V, Bifulco M, De Petrocellis L. The endocannabinoid system and its therapeutic exploitation. Nat Rev Drug Discov. 2004;3:771-84.

8. Fowler CJ. Possible involvement of the endocannabinoid system in the actions of three clinically used drugs. Trends Pharmacol Sci. 2004;25:59-61.

9. Cota D, Marsicano G, Tschöp M, et al. The endogenous cannabinoid system affects energy balance via central orexigenic drive and peripheral lipogenesis. J Clin Invest. 2003;112:423-31.

10. Ravinet Trillou C, Delgorge C, Menet C, et al. CB1 cannabinoid receptor knockout in mice leads to leanness, resistance to dietinduced obesity and enhanced leptin sensitivity. Int J Obes Relat Metab Disord. 2004;28:640-8.

11. Di Marzo V. The endocannabinoid system: its general strategy of action, tools for its pharmacological manipulation and potential therapeutic exploitation. Pharmacol Res. 2009;60:77-84.

12. Katona I, Freund TF. Endocannabinoid signaling as a synaptic circuit breaker in neurological disease. Nat Med. 2008;14:923-30.

13. Wilson RI, Nicoll RA. Endocannabinoid signaling in the brain. Science. 2002;296:678-82.

14. Matias I, Di Marzo V. Endocannabinoids and the control of energy balance. Trends Endocrinol Metab. 2007;18:27-37.

15. Zoerner AA, Gutzki FM, Batkai S, et al. Quantification of endocannabinoids in biological systems by chromatography and mass spectrometry: a comprehensive review from an analytical and biological perspective. Biochim Biophys Acta. 2011;1811:706-23.

16. • Fanelli F, Di Lallo VD, Belluomo I, et al. Estimation of reference intervals of five endocannabinoids and endocannabinoid related compounds in human plasma by two dimensional-LC/MS/MS. J Lipid Res. 2012;53:481-93. The authors established the reference intervals for circulating endocannabinoids in 121 healthy normal weight subjects. 
17. - Gatta-Cherifi B, Matias I, Vallée M, et al. Simultaneous postprandial deregulation of the orexigenic endocannabinoid anandamide and the anorexigenic peptide $\mathrm{YY}$ in obesity. Int $\mathrm{J}$ Obes. 2012;36:880-5. This article illustrates the relationship between feeding status and plasma endocannabinoid levels.

18. Zoerner AA, Batkai S, Suchy MT, et al. UPLC-MS/MS quantification of the endocannabinoids 2-arachidonoyl glycerol (2AG), 1-arachidonoyl glycerol (1AG), and anandamide in human plasma: minimization of matrix-effects, $2 \mathrm{AG} / 1 \mathrm{AG}$ isomerization and degradation by toluene solvent extraction. J Chromatogr B Analyt Technol Biomed Life Sci. 2012;883:161-71.

19. Blüher M, Engeli S, Klöting N, et al. Dysregulation of the peripheral and adipose tissue endocannabinoid system in human abdominal obesity. Diabetes. 2006;55:3053-60.

20. Gorzalka BB, Dang SS. Endocannabinoids and gonadal hormones: bidirectional interactions in physiology and behavior. Endocrinology. 2012;153:1016-24.

21. Paradisi A, Oddi S, Maccarrone M. The endocannabinoid system in ageing: a new target for drug development. Curr Drug Targets. 2006; 7:1539-52.

22. Monteleone P, Matias I, Martiadis V, et al. Blood levels of the endocannabinoid anandamide are increased in anorexia nervosa and in binge-eating disorder, but not in bulimia nervosa. Neuropsychopharmacology. 2005;30:1216-21.

23. Engeli S, Böhnke J, Feldpausch M, et al. Activation of the peripheral endocannabinoid system in human obesity. Diabetes. 2005;54:2838-43.

24. Matias I, Gonthier MP, Orlando P, et al. Regulation, function, and dysregulation of endocannabinoids in models of adipose and betapancreatic cells and in obesity and hyperglycemia. J Clin Endocrinol Metab. 2006;91:3171-80.

25. Matias I, Gonthier MP, Petrosino S, et al. Role and regulation of acylethanolamides in energy balance: focus on adipocytes and beta-cells. Br J Pharmacol. 2007;152:676-90.

26. Côté M, Matias I, Lemieux I, et al. Circulating endocannabinoid levels, abdominal adiposity and related cardiometabolic risk factors in obese men. Int J Obes. 2007;31:692-9.

27. • Di Marzo V, Verrijken A, Hakkarainen A, et al. Role of insulin as a negative regulator of plasma endocannabinoid levels in obese and nonobese subjects. Eur J Endocrinol. 2009;161:715-22. This article illustrates the inverse relationship between insulin sensitivity and endocannabinoids levels in humans.

28. Kopelman PG. Obesity as a medical problem. Nature. 2000;404:635-43.

29. Quercioli A, Pataky Z, Vincenti G, et al. Elevated endocannabinoid plasma levels are associated with coronary circulatory dysfunction in obesity. Eur Heart J. 2011;32:1369-78.

30. Jumpertz R, Wiesner T, Blüher M, et al. Circulating endocannabinoids and $\mathrm{N}$-acyl-ethanolamides in patients with sleep apnea-specific role of oleoylethanolamide. Exp Clin Endocrinol Diabetes. 2010;118:591-5.

31. - Jumpertz R, Guijarro A, Pratley RE, et al. Central and peripheral endocannabinoids and cognate acylethanolamides in humans: association with race, adiposity, and energy expenditure. J Clin Endocrinol Metab. 2011;96:787-91. The investigators demonstrated an opposite correlation between paired CSF and plasma endocannabinoids with metabolic markers.

32. - Matias I, Gatta-Cherifi B, Tabarin A, Clark S, Leste-Lasserre T, Marsicano $G$ et al.: Endocannabinoids measurement in human saliva as potential biomarker of obesity. PLoS One. 2012;7: e42399. The investigators demonstrated that endocannabinoids are quantifiable in human saliva.

33. Sipe JC, Waalen J, Gerber A, Beutler E. Overweight and obesity associated with a missense polymorphism in fatty acid amide hydrolase (FAAH). Int J Obes. 2005;29:755-9.

34. Bordicchia M, Battistoni I, Mancinelli L, et al. Cannabinoid CB1 receptor expression in relation to visceral adipose depots, endocannabinoid levels, microvascular damage, and the presence of the Cnr1 A3813G variant in humans. Metabolism. 2010;59:734-41.

35. Baye TM, Zhang Y, Smith E, et al. Genetic variation in cannabinoid receptor 1 (CNR1) is associated with derangements in lipid homeostasis, independent of body mass index. Pharmacogenomics. 2008;9:1647-56.

36. Aberle J, Flitsch J, Beck NA, et al. Genetic variation may influence obesity only under conditions of diet: analysis of three candidate genes. Mol Genet Metab. 2008;95:188-91.

37. - Sipe JC, Scott TM, Murray S, et al. Biomarkers of endocannabinoid system activation in severe obesity. PLoS One. 2010;20(1):e8792. This study demonstrated an association between severe obesity, FAAH polymorphism and plasma endocannabinoids levels.

38. Engeli S, Heusser K, Janke J, et al. Peripheral endocannabinoid system activity in patients treated with sibutramine. Obesity. 2008;16:1135-7.

39. Di Marzo V, Côté M, Matias I, et al. Changes in plasma endocannabinoid levels in viscerally obese men following a 1 year lifestyle modification programme and waist circumference reduction: associations with changes in metabolic risk factors. Diabetologia. 2009;52:213-7.

40. Artmann A, Petersen G, Hellgren LI, et al. Influence of dietary fatty acids on endocannabinoid and $\mathrm{N}$-acylethanolamine levels in rat brain, liver and small intestine. Biochim Biophys Acta. 2008;1781:200-12.

41. Gómez R, Navarro M, Ferrer B, et al. A peripheral mechanism for CB1 cannabinoid receptor-dependent modulation of feeding. $\mathrm{J}$ Neurosci. 2002;22:9612-7.

42. Kirkham TC, Williams CM, Fezza F, Di Marzo V. Endocannabinoid levels in rat limbic forebrain and hypothalamus in relation to fasting, feeding and satiation: stimulation of eating by 2 arachidonoyl glycerol. Br J Pharmacol. 2002;136:550-7.

43. Joosten MM, Balvers MG, Verhoeckx KC, Hendriks HF, Witkamp RF. Plasma anandamide and other $\mathrm{N}$-acylethanolamines are correlated with their corresponding free fatty acid levels under both fasting and non-fasting conditions in women. Nutr Metab. 2010;7:49.

44. - Monteleone P, Piscitelli F, Scognamiglio P, et al. Hedonic eating is associated with increased peripheral levels of ghrelin and the endocannabinoid 2-arachidonoyl-glycerol in healthy humans: a pilot study. J Clin Endocrinol Metab. 2012;97(6):E917-24. The investigators evaluated the relationship between hedonic eating and plasma endocannabinoids levels.

45. Yoshida R, Ohkuri T, Jyotaki M, et al. Endocannabinoids selectively enhance sweet taste. Proc Natl Acad Sci U S A. 2010;107:935-9. 